\title{
SIMPLICITY BIASES OPERATE AT ITEM AND SYSTEM LEVEL. AN ITERATED LEARNING STUDY
}

\author{
ALIKI PAPA $^{1}$, MIRIAM STADJE ${ }^{2}$, SIMON KIRBY ${ }^{2}$ AND MONICA TAMARIZ* \\ *Corresponding Author: M.Tamariz@hw.ac.uk \\ ${ }^{1}$ Psychology, Heriot-Watt University, Edinburgh, UK \\ ${ }^{2}$ CLE, PPLS, The University of Edinburgh, Edinburgh, UK
}

Cognitive biases, amplified over repeated cultural transmission, leave their mark on the structure of behaviour including language (Kirby et al., 2007). A key learning bias is the preference for simplicity - specifically, compressible representations (Kirby et al. 2015; Regier et al., 2015). The emergence of simplicity over cultural transmission has been experimentally attested: on one hand, individual items such as drawings become less complex graphically and increasingly recognizeable under the effects of schemas, or attractors (Bartlett, 1932; Sperber, 1996; Tamariz \& Kirby, 2015). On the other hand, sets of items become compressible when initially independent, idiosyncratic elements develop system-level properties such as compositionality in miniature artificial languages (Kirby et al., 2015) or systematicity across sets of melodies (Verhoef 2012), colour strings (Cornish et al. 2014), rhythmical structures (Ravignani et al. 2016), or visual patterns on a grid (Claidiere et al. 2014; Kempe et al. 2015).

This study systematically explores the simultaneous emergence of simplicity on multiple levels through cultural transmission in the graphical modality. Given the above results, we hypothesize, for repeatedly transmitted sets of drawings:

H1. The emergence of system-level properties leading to between-chain divergence and within-chain convergence of the drawings over generations. We expect chain-specific system-level properties such as a recogniseable chain-specific style, which contribute to set-wise simplicity, to emerge.

H2. The emergence of item-level properties. Compressibility will additionally be achieved at the item level through (a) progressive simplification of the 
graphical forms and (b) Increased recognizeability of individual drawings driven by schemas, or attractors.

We ran 10 transmission chains of 20 generations, with each participant reproducing 10 initially abstract drawings. Overall increase in set-wise simplicity, measured through an odd-one out selection task by naive participants, was not statistically significant. However, visual inspection found clear unique system properties, or style, emerging in several of the chains (H1). At the item level, graphical forms became significantly more compressible over generations (our complexity measure was scanned file size in KB) (H2a), and recognizeable schemas such as letters and numbers emerged throughout $(\mathrm{H} 2 \mathrm{~b})$.

Our results partially confirm the ubiquity of the simplicity bias operating in cultural transmission. They speak to theories that emphasise the role of learning biases (e.g. Kirby et al. 2015) or attraction (e.g. Sperber, 1996) in cultural evolution. Finally, they highlight that cognitive and learning biases can operate across cultural modalities (linguistic, musical, visual and graphical), supporting a domain-general view of the cultural evolutionary process.

\section{References}

Bartlett, F. C. (1932). Remembering. Cambridge: Cambridge University Press.

Claidière, N., Smith, K., Kirby, S. \& Fagot, J. (2014). Cultural evolution of systematically structured behaviour in a non-human primate. Proc. R. Soc. B 281, 20141541.

Cornish, H., Smith, K \& Kirby, S. (2013). Systems from sequences. Proceedings of the 35th Annual Cognitive Science Conference, 340-345.

Kempe, V., Gauvrit, N. \& Forsyth, D. (2015). Structure emerges faster during cultural transmission in children than in adults. Cognition 136, 247-254.

Kirby, S., Dowman, M. \& Griffiths, T. (2007). Innateness and culture in the evolution of language. PNAS, 104, 5241-45.

Kirby, S., Tamariz, M., Cornish, H. \& Smith, K. (2015). Compression and communication in the cultural evolution of linguistic structure. Cognition 141, 87-102.

Ravignani, A., Delgado, T. \& Kirby, S. (2016) Musical evolution in the lab exhibits rhythmic universals. Nature Human Behaviour, $1: 1$.

Regier, T., Kemp, C., Kay, P. (2015). Word meanings across languages support efficient communication. Handbook of Language Emergence, 87, 237-263.

Sperber, D. (1996). Explaining culture. Oxford: Blackwell.

Tamariz, M. \& Kirby, S. (2014). Culture: Copying, compression, and conventionality. Cognitive Science, 39, 171-183.

Verhoef, T. (2012). The origins of duality of patterning in artificial whistled languages. Language and Cognition, 4, 357-380. 